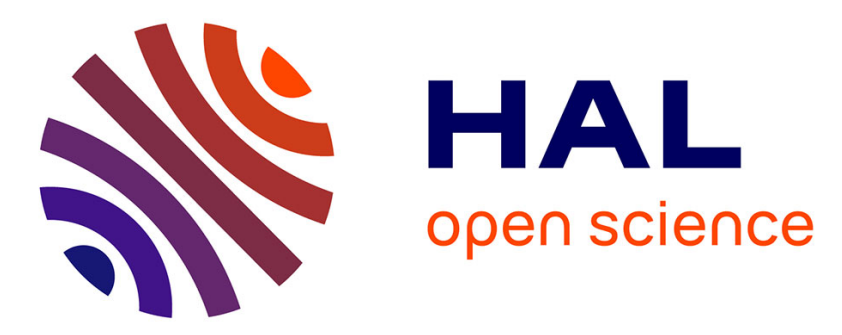

\title{
Mort soudaine et inattendue dans l'épilepsie : physiopathologie et données cliniques
}

\author{
M. Loizon, S. Rheims
}

\section{To cite this version:}

M. Loizon, S. Rheims. Mort soudaine et inattendue dans l'épilepsie: physiopathologie et données cliniques. Pratique Neurologique - FMC, 2019, 10, pp.131 - 135. 10.1016/j.praneu.2019.02.007 . hal-03486511

\section{HAL Id: hal-03486511 \\ https://hal.science/hal-03486511}

Submitted on 20 Dec 2021

HAL is a multi-disciplinary open access archive for the deposit and dissemination of scientific research documents, whether they are published or not. The documents may come from teaching and research institutions in France or abroad, or from public or private research centers.
L'archive ouverte pluridisciplinaire HAL, est destinée au dépôt et à la diffusion de documents scientifiques de niveau recherche, publiés ou non, émanant des établissements d'enseignement et de recherche français ou étrangers, des laboratoires publics ou privés.

\section{다)(1) $(5$}

Distributed under a Creative Commons Attribution - NonCommerciall 4.0 International 


\title{
Mort Soudaine et Inattendue dans l'Epilepsie : physiopathologie et données cliniques
}

Sudden and unexpected death in epilespsy: pathophysiology and clinical data

\author{
Marine Loizon ${ }^{1}$ et Sylvain Rheims ${ }^{1,2,3} *$
}

1. Service de Neurologie Fonctionnelle et Epileptologie, Hospices Civils de Lyon et Université de Lyon Lyon, Lyon, France

2. Centre de Recherche en Neuroscience de Lyon, INSERM U1028 / CNRS UMR5292, Lyon, France

3. Institut des Epilepsies (IDEE), Lyon, France

*Correspondance à :

Pr Sylvain Rheims, Service de Neurologie Fonctionnelle et Epileptologie, Hôpital Neurologique, 59 boulevard Pinel, 69677 Bron Cedex

sylvain.rheims@univ-lyon1.fr 


\section{Résumé}

Au cours des dix dernières années, cette problématique des morts subites et inattendues dans l'épilepsie (SUDEP) s'est progressivement imposée comme l'une des inquiétudes principales des patients, familles ou médecins concernés par l'épilepsie. A l'échelle de la population souffrant d'épilepsie, l'incidence des SUDEP est de 0,58 (0,31-1,08) / 1000 patients-années mais s'élève à 5/1000 patients-années en cas de pharmacorésistance. La présence et la fréquence des crises tonico-cloniques généralisées représentent le principal facteur de risque. Bien que les mécanismes physiopathologiques exacts qui mènent à la SUDEP demeurent inconnus, la plupart des données expérimentales et cliniques suggèrent le rôle prédominant de la dysfonction respiratoire post-ictale. Il n'existe à ce jour aucun traitement préventif spécifique des SUDEP. Actuellement, la seule prise en charge thérapeutique ayant démontré une efficacité dans la prévention des SUDEP reste l'optimisation du traitement antiépileptique. L'information vis à vis du risque, souvent souhaitée par les patients et leur famille, est à élément à prendre dans la prise en charge, en particulier en cas de crises tonicocloniques généralisées fréquentes.

Mots clés : Epilepsie, SUDEP, crises tonico-cloniques,

\section{$\underline{\text { Abstract }}$}

Over the past ten years, the issue of sudden and unexpected death in epilepsy (SUDEP) has gradually emerged as one of the main concerns of patients, families and physicians concerned with epilepsy. At the population level, the incidence of SUDEP is 0.58 (0.31-1.08) / 1000 patient-years but rises to 5/1000 patient-years in patients with drug-resistant epilepsy. The presence and rate of generalized tonic-clonic seizures is the main risk factor. Although the exact pathophysiological mechanisms leading to SUDEP remain unknown, most experimental and clinical data suggest the predominant role of post-ictal respiratory dysfunction. To date, there is no specific preventive treatment for SUDEP. Currently, the only therapeutic management that has demonstrated efficacy in the prevention of SUDEP remains the optimization of antiepileptic treatment. Risk information, often desired by patients and their families, is a key element in the management, especially in the case of frequent generalized tonic-clonic seizures.

Keywords : Epilepsy, SUDEP, generalized tonic-clonic seizures 
Parmi les 50 à 60 millions de personnes souffrant d'épilepsie dans le monde, environ un tiers est confronté à une résistance au traitement, les crises n'étant pas maîtrisées malgré le choix et l'utilisation appropriés des médicaments antiépileptiques (1). Les patients souffrant d'épilepsie pharmacorésistante ont un taux de mortalité environ cinq fois plus élevé que dans la population générale (2). Parmi les causes de décès prématurés chez les patients épileptiques, la mort subite et inattendue dans l'épilepsie (SUDEP) représente une cause majeure. La SUDEP correspond à un décès non traumatique et sans noyade chez les patients souffrant d'épilepsie, non lié à un état de mal épileptique documenté et pour lequel l'examen postmortem ne révèle aucune cause toxicologique ou anatomique explicative (2). Au cours des dix dernières années, cette problématique des SUDEP s'est progressivement imposée comme l'une des inquiétudes principales des patients, familles ou médecins concernés par l'épilepsie. Au-delà des aspects scientifiques visant à mieux comprendre la physiopathologie des SUDEP et à mieux identifier les patients à risque, la communauté épileptologique s'est organisée autour d'une réflexion vis à vis de l'information à donner concernant le risque de SUDEP et à l'accompagnement des familles endeuillées, en particulier au travers des actions du Réseau Sentinelle Mortalité Epilepsie (RSME) coordonné par le Dr Marie-Christine Picot sous l'égide de la Ligue Française contre l'Epilepsie et avec le soutien de la Fondation Française pour la Recherche sur l'Epilepsie (http://www.lfce.fr/Le-Reseau-Sentinelle-MortaliteEpilepsie-RSME_a46.html).

\section{Epidémiologie et facteurs de risque}

A l'échelle de la population souffrant d'épilepsie, l'incidence des SUDEP est de 0,58 (0,311,08) / 1000 patients-années (3). L'incidence apparait plus importante chez l'adulte $(1,2$ (0,642,32)) que chez l'enfant $(0,22(0,16-0,31))(3)$. Néanmoins, ce constat a été récemment remis en cause à travers deux études aux méthodologies solides réalisées respectivement au Canada et en Suède et rapportant une incidence chez l'enfant proche de celle de l'adulte $(4,5)$. En revanche, toutes les données sont extrêmement convergentes quant à la variation du risque de SUDEP selon le type d'épilepsie, avec un risque significativement plus élevé chez les patients atteints d'épilepsie résistante aux médicaments (3). L'incidence de SUDEP est ainsi estimée à 5/1000 patients-années en cas de pharmacorésistance (3). De même, une étude a révélé que jusqu'à $20 \%$ des patients atteints d'épilepsie pharmacorésistante ayant débuté dans l'enfance mourront d'une SUDEP à l'âge de 45 ans (6), avec un risque particulièrement marqué dans des épilepsies complexes associées à des troubles du développement cognitif. 
D'autres facteurs de risque de SUDEP ont été individualisés (2). La présence et la fréquence des crises tonico-cloniques généralisées (CTCG), qu'elles soient primaires ou secondairement généralisées, représentent le principal facteur de risque, avec un rapport de cotes $>15$ pour les patients ayant trois CTCG ou plus par mois (7). Quelques autres facteurs de risque se sont révélés significatifs, mais avec des rapports de cotes $<2$, y compris le sexe masculin, l'âge d'apparition de l'épilepsie <16 ans, la durée de l'épilepsie >15 ans et la polythérapie (8). Cependant, lorsque des ajustements ont été faits pour tenir compte du nombre de SUDEP, ni la polythérapie ni l'utilisation d'antiépileptiques spécifiques comme la lamotrigine ou la carbamazépine n'étaient associées à un risque accru de SUDEP (7). Il est intéressant de noter qu'il a également été suggéré que l'apparition nocturne de CTCG pourrait être plus à risque de SUDEP, une observation qui pourrait refléter soit des différences circadiennes dans la réponse cérébrale aux CTCG pendant le sommeil, avec un risque accru de dysfonctionnement neurovégétatif pendant la nuit, soit une supervision plus efficace et une réanimation plus rapide pendant le jour (9).

\section{Physiopathologie}

Les mécanismes physiopathologiques exacts qui mènent à la SUDEP demeurent inconnus (2). Cependant, comme nous le verrons plus loin, la plupart des données probantes appuient le rôle prédominant de la dysfonction respiratoire (2). La possibilité que l'événement primaire soit une dysrythmie cardiaque ictale ou un autre processus autonome ou central semble moins probable ou pourrait expliquer une minorité de SUDEP (2). Par exemple, l'asystolie ictale, définie comme une pause sinusale d'au moins 3 secondes pendant une crise, dure habituellement 60 secondes et est spontanément réversible $(10,11)$.

Ainsi, les données expérimentales et cliniques suggèrent fortement que la plupart des SUDEP résultent d'une dysfonction respiratoire post-critique évoluant vers une apnée terminale, suivie plus tard d'un arrêt cardiaque.

- L'apnée était la principale cause de décès chez plusieurs modèles animaux de SUDEP : une étude chez les ovins et d'autres chez les rongeurs, y compris les souris soumises à des crises audiogènes (souris DBA/2 et DBA/1) ou chez les souris Kcna1-null (2). De plus, dans un modèle murin du syndrome de Dravet (Scn1aR1407X/+), une épilepsie sévère débutant chez l'enfant, la SUDEP résultait également d'une apnée 
centrale primaire, qui causait une bradycardie, probablement par les effets directs de l'hypoxémie sur le muscle cardiaque (12).

- Chez les patients dont la SUDEP s'était produite en hospitalisation au cours d'un monitoring vidéo-EEG prolongé, une analyse détaillée du matériel vidéo, de l'EEG et de l'ECG au moment des arrêts cardiorespiratoires a pu être réalisée. Celle-ci mettait en évidence une apnée centrale post-critique dans toutes les SUDEP (9). Ce dysfonctionnement respiratoire central survenait généralement entre 1 et 3 minutes après la fin d'une crise tonico-clonique généralisée, en association avec une bradycardie sévère ou une asystolie transitoire (9).

Le rôle clé du dysfonctionnement respiratoire dans la cascade d'événements qui mène à la SUDEP a été renforcé par les nombreuses données démontrant que l'épilepsie réfractaire est associée à une altération chronique de la régulation respiratoire :

- Dans des modèles établis de crises convulsives chez le rat, plusieurs études (13) ont observé que les rats, qui souffrent de crises spontanées chroniques, développent également des troubles respiratoires. Conformément au rôle clé de la sérotonine dans la régulation de la respiration, il a été suggéré à plusieurs reprises que cette dysfonction respiratoire liée aux crises, et éventuellement la SUDEP, pourrait être liée au dysfonctionnement des neurones sérotoninergiques $(14,15)$.

- Plus important encore, une étude anatomopathologique des structures du tronc cérébral, et particulièrement du bulbe, comparant témoins, patients décédés de SUDEP et patients souffrant d'épilepsie décédés d'une autre cause, a récemment confirmé l'existence d'altérations spécifiques des structures et voies moléculaires impliquées dans le contrôle respiratoire (16). Ce travail montrait en particulier une perte en neurones exprimant le récepteur à la neurokinine 1 dans le complexe pré-Bötzinger et des modifications de la voie sérotoninergique dans le raphé bulbaire (16).

- En parallèle, il a été démontré que les deux tiers des patients développeront une désaturation transitoire en oxygène $<90 \%$ pendant au moins une de leurs crises enregistrées (17). Dans des crises strictement focales, une apnée survient dans $33 \%$ des crises $(17,18)$ et peut entraîner une désaturation en oxygène allant jusqu'à 50\% (17). Dans les crises avec une évolution secondairement généralisée, une désaturation <90\% prolongée plus de 30 secondes après la fin de la crise est observée dans $84 \%$ des crises (19). Il est important de noter ici que la durée de la désaturation dans les CTCG partage 
avec les apnées observées dans les crises strictement focales un lien significatif avec la localisation temporale de la zone épileptogène $(18,19)$, suggérant la mise en jeu spécifique de réseaux impliqués dans le contrôle respiratoire. Néanmoins, la relation exacte entre la survenue et/ou la gravité d'une apnée transitoire au cours de crises focales ou généralisées et le risque de SUDEP n'a pas encore été établie formellement. Cette question importante est l'objectif principal d'une étude prospective multicentrique française, basée sur le Réseau National de Recherche sur les prédicteurs des SUDEP (PHRC National REPOMSE 2009). Celle-ci a inclus entre 2011 et 2015, 1066 patients ayant bénéficié d'un monitoring vidéo-EEG préchirurgical dont le suivi est en cours afin d'identifier prospectivement les cas de SUDEP et d'évaluer si les troubles respiratoires per-ictaux étaient plus fréquents chez ces derniers.

Un autre facteur associé à la SUDEP est la suppression généralisée post-ictale de l'EEG (PGES), définie comme l'absence généralisée d'activité électroencéphalographique $>10 \mu \mathrm{V}$ débutant dans les 30 secondes après la fin de la crise (20). Cependant, l'importance pathophysiologique exacte de la PGES reste controversée (2). Plusieurs données mettent en évidence un lien entre PGES et trouble respiratoire post-ictal $(19,21)$, suggérant que la PGES ne pourrait être qu'un marqueur ancillaire d'une dysfonction profonde des structures impliquées dans le contrôle neurovégétatif.

\section{Prévention et prise en charge du risque}

\section{Prévention spécifique?}

Il n'existe à ce jour aucun traitement préventif spécifique des SUDEP. Bien que la relation exacte entre l'apparition et /ou la sévérité des apnées liées aux crises et le risque de SUDEP reste à établir formellement, la focalisation sur la dysfonction respiratoire liée à la crise apparaît actuellement comme la stratégie la plus pertinente pour développer de nouvelles approches thérapeutiques (22).

Ainsi, tous les essais contrôlés randomisés achevés et/ou en cours dans le domaine des SUDEP visent à évaluer l'efficacité d'une intervention pharmacologique spécifique dans la réduction de la dysfonction respiratoire centrale post-ictale chez les patients souffrant d'épilepsie (2). La première voie thérapeutique qui a été envisagée a été celle de l'effet préventif d'un traitement sérotoninergique au long cours sur les apnées centrales postcritiques (22). Des deux études ont été conduites mais ont été confrontées à des difficultés 
importantes de recrutement des patients. Seul le résultat de l'une d'entre elles pourrait être d'intérêt mais son analyse reste en cours. Parallèlement à l'hypothèse de la sérotonine, une étude française évalue également l'intérêt potentiel de cibler la voie des opioïdes dans un essai randomisé multicentrique qui vise à évaluer l'efficacité d'un antagoniste opiö̈de injectable (Naloxone) versus placebo sur la réduction de la sévérité de la dysfonction respiratoire centrale secondaire à une crise tonico-clonique généralisée (23).

Il est important de noter que, même si l'une de ces études montre des résultats positifs, elle représente seulement une étape initiale dans la prévention de SUDEP. L'étape suivante et la plus importante sera de démontrer que l'efficacité de l'intervention thérapeutique ne se limite pas à la réduction de la dysfonction respiratoire centrale post-ictale, mais que celle-ci prévient les SUDEP. Cela implique d'entreprendre une étude à grande échelle chez les patients ambulatoires pour tester l'impact du traitement sur le risque de SUDEP. Cependant, la conception d'un essai clinique visant la prévention des SUDEP pose d'importants problèmes méthodologiques, en lien avec la taille de la population nécessaire pour démontrer sur 1 an une baisse significative de risque chez les patients souffrant d'épilepsie réfractaire où l'incidence attendue des SUDEP est $0,5 \%$ par an (24). Au-delà de l'identification de cibles thérapeutiques spécifiques, le développement de traitements préventifs des SUDEP passera obligatoirement par l'amélioration de notre connaissance des facteurs de risques afin d'identifier des populations à risque beaucoup plus élevé de SUDEP (24).

\section{Optimiser la prise en charge pour réduire le risque de SUDEP ?}

Actuellement, la seule prise en charge thérapeutique ayant démontré une efficacité dans la prévention des SUDEP reste l'adjonction d'un traitement antiépileptique à doses efficaces chez un patient souffrant d'une épilepsie pharmacorésistante. A partir d'approches méthodologiques de méta-analyses incluant 112 essais thérapeutiques ayant évalué un médicament antiépileptique contre placebo lors d'un essai de phase III, il a ainsi été montré que le risque de SUDEP était sept fois moins important dans les groupes recevant la molécule active que dans ceux recevant le placebo (25).

L'impact exact de la chirurgie de l'épilepsie sur le risque de SUDEP reste à préciser. La difficulté d'analyse des données existantes réside principalement dans la problématique du risque de SUDEP chez les patients opérés mais non totalement libres de crise (26). Néanmoins, compte tenu de la physiopathologie des SUDEP et leur lien avec la survenue de 
crises, il est intuitif d'envisager que chez les patients pour lesquels la guérison est réellement obtenue, le risque de SUDEP disparaisse. Au-delà de la chirurgie de l'épilepsie, des données récentes ont suggéré que la mise en place d'une stimulation du nerf vague pourrait s'associer sur le long terme à une diminution du risque de SUDEP (27).

Une mauvaise observance thérapeutique fait partie des facteurs de risques envisagés pour les SUDEP (2). Dans ce contexte, même si non démontré, favoriser l'adhésion à la prise en charge doit être considéré comme un aspect primordial de la prévention. Divers éléments pourraient favoriser l'adhésion. On citera ici en particulier la question du nombre de prises quotidiennes de traitement pour laquelle le bénéfice semble indéniable entre 1 à 2 prises/j et 3 ou 4 prises quotidiennes (28). Par ailleurs, même si le bénéfice à l'échelle de l'ensemble des patients des programmes d'éducation thérapeutique et/ou des accompagnements par des infirmières cliniciennes spécialisées en épilepsie reste à évaluer formellement (29), en particulier dans le cadre du système de soins français, il est certain que ces prises en charge ne doivent pas être négligées chez des patients qui seraient en difficulté avec le suivi du traitement.

\section{Informer patients et familles}

La difficulté à individualiser le risque de SUDEP a souvent été mis en perspective avec la difficulté à donner une information qui serait inutilement anxiogène parce que le risque est en réalité très faible chez le patient et/ou parce qu'aucune prise en charge spécifique ne peut être proposée pour diminuer significativement le risque. Pourtant la discussion du risque de SUDEP est devenue une obligation médico-légale dans certains pays (30,31). De plus, les inquiétudes vis à vis de la discussion du risque de SUDEP sont avant tout liées au ressenti potentiel du patient et de son entourage face à cette information. Elles sont néanmoins en partie infondées, car si certains patients rapportaient s'être sentis anxieux transitoirement, aucun ne rapportait de complication psychologique ou psychiatrique au long cours suite à l'annonce (32). Aussi, en épileptologie pédiatrique, ceci s'associait à une augmentation de la surveillance de l'épilepsie et de l'observance du traitement par les parents dans environ un tiers des cas (33). Surtout, toutes les études qui ont essayé d'évaluer le souhait des patients et de leur entourage convergent vers une volonté de leur part d'être informés du risque de SUDEP (32).

Par ailleurs, ces éléments sont également à mettre en perspective avec l'éventualité que l'entourage puisse être acteur de la prévention. En effet, les données rapportent une 
diminution du risque de SUDEP chez les patients bénéficiant d'une surveillance nocturne (34). Aussi, l'observation qu'aucun patient ayant pu bénéficier d'une réanimation très précoce lors d'un arrêt cardio-respiratoire post-critique survenu au cours d'une vidéo-EEG (9) ne soit décédé pose la question des organisations à domicile ou en institution permettant à un tiers d'intervenir rapidement. De même, les données suggérant que l'administration précoce d'oxygène au cours d'une CTCG permette de prévenir la survenue d'une PGES (21) et de réduire la durée de l'hypoxie post-critique (19) pourraient laisser envisager une place thérapeutique pour l'oxygénothérapie préventive. Ces aspects pourraient en particulier bénéficier des développements attendus des systèmes de détection de crises (35) .

En cloncusion, la question du principe de l'information vis à vis du risque de SUDEP ne semble plus faire discussion. Il reste néanmoins indispensable de l'aborder de manière humaine, respectueuse et en écoutant ce que souhaite le patient. Une approche modulable pourrait ainsi s'envisager dans laquelle la porte à la discussion sur la mortalité dans l'épilepsie, dont les SUDEP, serait ouverte de manière systématique mais en adaptant également toujours l'évolution de la conversation. En effet, le détail des informations serait donné en fonction de ce que semble souhaiter le patient ou sa famille et/ou du niveau de risque auquel le patient est exposé. Par ailleurs, si cette discussion va pointer un risque non négligeable chez certains patients, en particulier ceux présentant des crises tonico-cloniques généralisées, elle peut aussi être l'occasion de rassurer sur la faiblesse du risque d'autres patients qui auraient eu accès à une information incomplète, par exemple à travers internet.

\section{Liens d'intérêts}

ML n'a pas de lien d'intérêt.

SR a reçu des honoraires d'orateur et/ou de consultant des laboratoires EISAI, UCB Pharma, Livanova, GW Pharma, Idiorsia et Novartis. 


\section{Points essentiels}

- La présence et la fréquence des crises tonico-cloniques généralisées représentent le principal facteur de risque de SUDEP

- II n'existe à ce jour aucun traitement préventif spécifique des SUDEP

- L'adjonction d'un traitement antiépileptique à doses efficaces chez un patient souffrant d'une épilepsie pharmacorésistante diminue significativement le risque de SUDEP

- Les études convergent vers un souhait des patients et de leur famille d'être informés du risque de SUDEP

- En permettant une intervention post-ictale, la supervision nocturne et la détection des crises pourraient représenter un élément important de la prévention 


\section{Références}

1. Kwan P, Arzimanoglou A, Berg AT, et al. Definition of drug resistant epilepsy: consensus proposal by the ad hoc Task Force of the ILAE Commission on Therapeutic Strategies. Epilepsia 2010;51:1069-77.

2. Devinsky O, Hesdorffer DC, Thurman DJ, Lhatoo S, and Richerson G. Sudden unexpected death in epilepsy: epidemiology, mechanisms, and prevention. Lancet Neurol 2016;15:1075-88.

3. Harden C, Tomson T, Gloss D, et al. Practice guideline summary: Sudden unexpected death in epilepsy incidence rates and risk factors: Report of the Guideline Development, Dissemination, and Implementation Subcommittee of the American Academy of Neurology and the American Epilepsy Society. Neurology 2017;88:1674-80.

4. Keller AE, Whitney R, Li SA, Pollanen MS, and Donner EJ. Incidence of sudden unexpected death in epilepsy in children is similar to adults. Neurology 2018

5. Sveinsson O, Andersson T, Carlsson S, and Tomson T. The incidence of SUDEP: A nationwide population-based cohort study. Neurology 2017

6. Sillanpaa M and Shinnar S. Long-term mortality in childhood-onset epilepsy. N Engl J Med 2010;363:2522-29.

7. Hesdorffer DC, Tomson T, Benn E, et al. Do antiepileptic drugs or generalized tonicclonic seizure frequency increase SUDEP risk? A combined analysis. Epilepsia 2012;53:249-52.

8. Hesdorffer DC, Tomson T, Benn E, et al. Combined analysis of risk factors for SUDEP. Epilepsia 2011;52:1150-59.

9. Ryvlin P, Nashef L, Lhatoo SD, et al. Incidence and mechanisms of cardiorespiratory arrests in epilepsy monitoring units (MORTEMUS): a retrospective study. Lancet Neurol 2013;12:966-77.

10. Rugg-Gunn FJ, Simister RJ, Squirrell M, Holdright DR, and Duncan JS. Cardiac arrhythmias in focal epilepsy: a prospective long-term study. Lancet 2004;364:2212-19.

11. Schuele SU, Bermeo AC, Alexopoulos AV, et al. Video-electrographic and clinical features in patients with ictal asystole. Neurology 2007;69:434-41.

12. Kim Y, Bravo E, Thirnbeck CK, et al. Severe peri-ictal respiratory dysfunction is common in Dravet syndrome. J Clin Invest 2018;128:1141-53.

13. Villiere SM, Nakase K, Kollmar R, Silverman J, Sundaram K, and Stewart M. Seizureassociated central apnea in a rat model: Evidence for resetting the respiratory rhythm and activation of the diving reflex. Neurobiol Dis 2017;101:8-15.

14. Buchanan Gordon F., Murray Nicholas M., Hajek Michael A., and Richerson George B. Serotonin neurones have anti-convulsant effects and reduce seizure-induced mortality: Seizure-induced death in. J Physiol 2014;592:4395-410.

15. Zhan Q, Buchanan GF, Motelow JE, et al. Impaired Serotonergic Brainstem Function during and after Seizures. J Neurosci 2016;36:2711-22.

16. Patodia S, Somani A, O'Hare M, et al. The ventrolateral medulla and medullary raphe in sudden unexpected death in epilepsy. Brain 2018;141:1719-33.

17. Bateman LM, Li CS, and Seyal M. Ictal hypoxemia in localization-related epilepsy: analysis of incidence, severity and risk factors. Brain 2008;131:3239-45.

18. Lacuey Nuria, Zonjy Bilal, Hampson Johnson P., et al. The incidence and significance of periictal apnea in epileptic seizures. Epilepsia 2018;59:573-82.

19. Rheims S, Mercedes B, Alexandre V, et al. Hypoxemia following generalized convulsive seizures: risk factors and impact of oxygen therapy. Neurology 2018; In Press 
20. Lhatoo Samden D., Faulkner Howard J., Dembny Krystina, Trippick Kathy, Johnson Claire, and Bird Jonathan M. An electroclinical case-control study of sudden unexpected death in epilepsy. Ann Neurol. 2010;68:787-96.

21. Alexandre V, Mercedes B, Valton L, et al. Risk factors of postictal generalized EEG suppression in generalized convulsive seizures. Neurology 2015;85:1598-603.

22. Ryvlin P, Nashef L, and Tomson T. Prevention of sudden unexpected death in epilepsy: a realistic goal. Epilepsia 2013;54 Suppl 2:23-28.

23. Rheims S, Valton L, Michel V, et al. Efficacy of naloxone in reducing postictal central respiratory dysfunction in patients with epilepsy: study protocol for a double-blind, randomized, placebo-controlled trial. Trials 2016;17:529.

24. Richerson GB, Boison D, Faingold CL, and Ryvlin P. From unwitnessed fatality to witnessed rescue: Pharmacologic intervention in sudden unexpected death in epilepsy. Epilepsia 2016;57 Suppl 1:35-45.

25. Ryvlin P, Cucherat M, and Rheims S. Risk of sudden unexpected death in epilepsy in patients given adjunctive antiepileptic treatment for refractory seizures: a meta-analysis of placebo-controlled randomised trials. Lancet Neurol 2011;10:961-68.

26. Seymour N, Granbichler CA, Polkey CE, and Nashef L. Mortality after temporal lobe epilepsy surgery. Epilepsia 2011

27. Ryvlin P, So EL, Gordon CM, et al. Long-term surveillance of SUDEP in drug-resistant epilepsy patients treated with VNS therapy. Epilepsia 2018;59:562-72.

28. Bialer M. Extended-release formulations for the treatment of epilepsy. CNS Drugs 2007;21:765-74.

29. da Mota Gomes M, Navarro T, Keepanasseril A, Jeffery R, and Haynes RB. Increasing adherence to treatment in epilepsy: what do the strongest trials show. Acta Neurol Scand 2017;135:266-72.

30. NICE. The diagnosis and management of the epilepsies in adults and children in primary and secondary care. Available at: http://guidance.nice.org.uk/CG137/NICEGuidance/pdf/English. Accessed 23/02/2017.

31. SIGN. Diagnosis and management of epilepsy in adults. Available at: http://sign.ac.uk/pdf/SIGN143.pdf.

32. Tonberg A, Harden J, McLellan A, Chin RF, and Duncan S. A qualitative study of the reactions of young adults with epilepsy to SUDEP disclosure, perceptions of risks, views on the timing of disclosure, and behavioural change. Epilepsy Behav 2015;42:98106.

33. Gayatri NA, Morrall MC, Jain V, Kashyape P, Pysden K, and Ferrie C. Parental and physician beliefs regarding the provision and content of written sudden unexpected death in epilepsy (SUDEP) information. Epilepsia 2010;51:777-82.

34. Langan Y, Nashef L, and Sander JW. Sudden unexpected death in epilepsy: a series of witnessed deaths. J Neurol Neurosurg Psychiatry 2000;68:211-13.

35. Van de Vel A, Cuppens K, Bonroy B, et al. Non-EEG seizure detection systems and potential SUDEP prevention: State of the art: Review and update. Seizure 2016;41:14153. 